\title{
A 3D microfluidic device fabrication method using thermopress bonding with multiple layers of polystyrene film
}

\author{
Yuanzhi Cao, Jacob Bontrager-Singer, Likun Zhu*
}

Department of Mechanical Engineering, Indiana University - Purdue University Indianapolis, Indianapolis, IN 46202, USA

*Corresponding Author:

Likun Zhu

Department of Mechanical Engineering

Indiana University Purdue University Indianapolis

723 W. Michigan Street, Room SL 260 L

Indianapolis, IN 46202

Phone: 1-317-274-4887

Fax: 1-317-274-9744

Email:likzhu@iupui.edu

This is the author's manuscript of the article published in final edited form as:

Cao, Y., Bontrager-Singer, J., \& Zhu, L. (2015). A 3D microfluidic device fabrication method using thermopress bonding with multiple layers of polystyrene film. Journal of Micromechanics and Microengineering, 25(6), pp. 65005-65014. http://dx.doi.org/10.1088/0960-1317/25/6/065005 


\begin{abstract}
In this paper, we present a fabrication method that is capable of making three dimensional (3D) microfluidic devices with multiple layers of homogeneous polystyrene (PS) film. PS film was chosen as primary device material due to its advantageous features for microfluidics applications. Thermopress is used as bonding method because it provides sufficient bonding strength while requires no heterogeneous bonding materials. By aligning and sequentially stacking multiple layers ( 3 to 20) of patterned PS film which were achieved by a craft cutter, complicated 3D structured microfluidic devices can be fabricated by multiple steps of thermopress bonding. The smallest feature can be achieved in this method is around $100 \mu \mathrm{m}$, which is limited by the resolution of the cutter $(25 \mu \mathrm{m})$ as well as the thickness of the PS films. Bonding characteristics of PS films are given in this paper, including PS film bonding strength test, bonding precision assessment, and PS surface wettability manipulation. To demonstrate the capability of this method, the design, fabrication and testing results of a $3 \mathrm{D}$ interacting L-shaped passive mixer are presented in the paper.
\end{abstract}

Keywords: Microfluidics, Digital craft cutter, Polystyrene film, Thermopress bonding 


\section{Introduction}

Microfluidics is emerging as a promising direction in chemical and biological applications. In the past decade, lots of research work has been done in the related areas [1-8]. Some of the previous microfluidic devices are based on sophisticated fabrication processes, including photolithography technology on silicon substrates [9], hot embossing and injection molding on polymer materials [10], soft lithography using Poly(dmethylsiloxane) (PDMS) casting [11], and direct machining using CNC milling machine [12]. These fabrication methods are generally capable of achieving high quality devices, yet they also require expensive equipment and long fabrication period.

As for scientific lab researchers who need to constantly adjust their designs, a microfluidic device fabrication method that is time and cost efficient while capable of obtaining reasonable precision is preferred. In searching for the answer to the above question, one of the solutions led to the emergence of film-based polymer microfluidic devices. In the publication of Yuen et al., they have described the fabrication process using a desktop digital craft cutter to create multiple patterned layers of polymer films. The patterned layers are then assembled and bonded together into a 3D microfluidic device by double-sided pressure sensitive adhesive (PSA) tapes [13]. This method is easy to implement and can reach a feature resolution as high as $200 \mu \mathrm{m}$. However, the use of PSA tapes in the device bonding process becomes a major problem of the method, because it creates a non-uniform channel side wall property. In addition, the PSA surface is hydrophobic and it is not desired in microfluidics applications.

In order to fabricate devices with uniform channel surface property, the use of homogeneous film materials is highly preferred. To achieve direct bonding between polymer films, thermopress bonding method was used in this study. The thermo bonding method has been used to fabricate microfluidic devices on different polymer substrates, such as Poly(methylmethacrylate) (PMMA), Polycarbonate (PC), Polyimide (PI), Polyethylene (PE), Poly(ethylene terephthalate) (PET), and Polystyrene (PS) [14-17]. Among polymer film materials, PS possesses advantageous features in microfluidics applications, because it is 
commonly available, highly transparent, biochemically compatible, and it has good material rigidity [18]. Most importantly, its surface wettability can be easily manipulated by oxygen plasma treatment $[19,20]$. Despite all these appealing characteristics, however, the thermopress bonding between PS films has rarely been utilized, and not enough attention has been received to push the research further into device applications.

Therefore, the purpose of this paper is to introduce a microfluidic device fabrication method based on uniform PS films using a digital craft cutter and thermopress bonding method. Different from previous publications regarding PS-based microfluidic devices and thermopress bonding, which usually include other structural materials like silicon, stainless steel, or other polymers, this paper focuses on using only patterned PS films from a digital craft cutter to form the complete structure of an integrated microfluidic device. This enables the device of being miniaturized and homogeneous while capable of being fabricated with low cost and short period that will fit a variety of applications. It is also noticed that the publication of Chen et al, has demonstrated a PS-based microfabrication method for microfluidics applications [21]. They used cutter to cut grooves on PS films, and bond them by heating to $160^{\circ} \mathrm{C}$. The groove channels will become deeper and thinner upon heating, which makes very small channel dimension possible. Our method, on the other hand, is more focused on bonding the PS films under the glass transition temperature of PS $\left(95^{\circ} \mathrm{C}\right)$, therefore maintaining the integrity of the channel dimension as designed.

In the following parts of the paper, bonding characterization of PS films will be given first, including the thermopress bonding strength test, bonding feature resolution and deformation examination, and PS surface wettability manipulation demonstration. The paper will then introduce our device fabrication method by presenting a 3D L-shaped microfluidic passive mixer as a demonstrating example. The mixer is achieved by sequentially stacking and aligning 14 layers of patterned PS films $(125 \mu \mathrm{m})$ with multiple steps of thermopress bonding. The detailed information about the device design, fabrication, and test results will be given. 


\section{Experimental}

\subsection{Material preparation}

The essential of this fabrication method is to use hot press to bond multiple layers of patterned PS film (Goodfellow, Coraopolis, PA) into a microfluidic device with internal 3D microchannels. The schematic of the bonding process is illustrated in Fig.1. A hydraulic hot press (Carver, Wabash, IN) was used in this bonding process. As shown in Fig.1 (a), under the applied pressure and temperature for a certain amount of time, the interface of PS to PS will be bonded together through molecular fusion transfer. To achieve uniform bonding results, two square glass plates $(54 \mathrm{~mm} \times 54 \mathrm{~mm} \times 6.35 \mathrm{~mm}$, McMaster, Chicago, IL) were used to sandwich the bonded PS layers. Also, it is important to align the patterned layers before bonding. This can be done by designing alignment features on each pattern and use pins to secure the alignment, as shown in Fig.1 (b). The patterns of the film were obtained by a digital craft cutter (FC2250-60 VC, Graphtec, Santa Ana, CA) fromcomputer-aided design (CAD) drawings. The internal 3D structured channel is made possible by the layer pattern as well as the film thickness, as shown in Fig.1 (c).

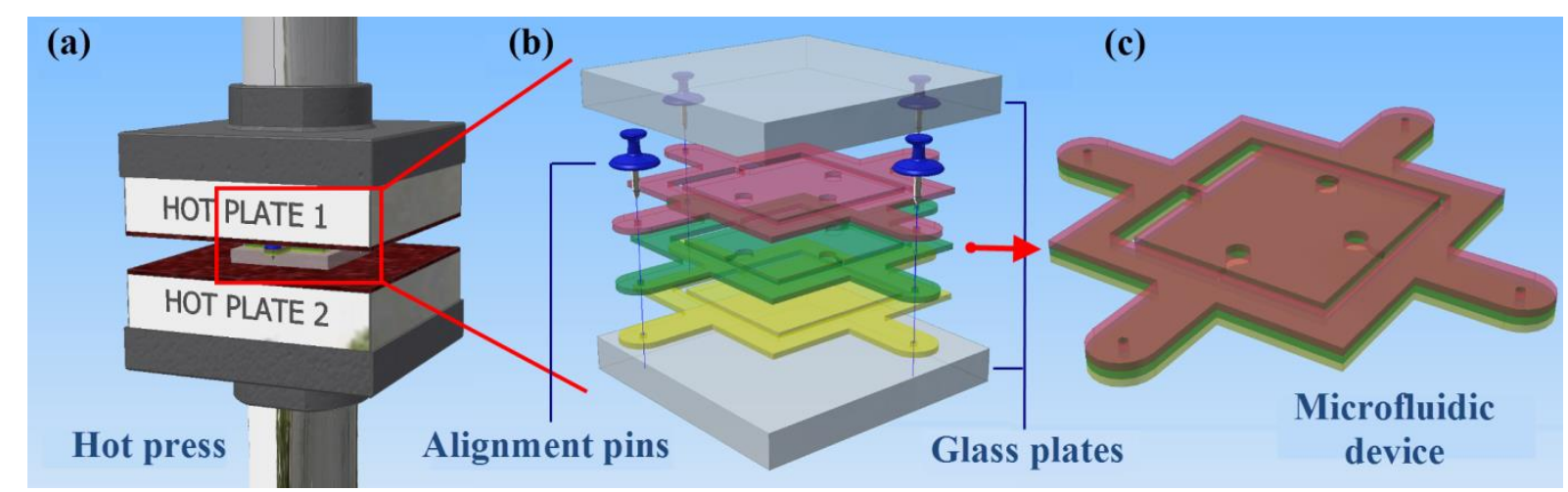

Fig. 1 The process overview of PS film-based microfluidic device fabrication method. (a) Thermopress bonding method by a hot press. (b) Patterned PS films sandwiched by glass plates and aligned by pins. (c) Finished film-based device by thermopress bonding.

The thermopress bonding of PS to PS is sensitive to surface cleanness. Contaminations like finger prints, water trace, and fiber dust on the surface will increase the chance of regional bonding defects that will cause critical bonding failure. Therefore, it is important to carefully prepare the surface before bonding. The surface preparation process is as follow: 
Rinse in isopropyl alcohol (IPA) for $1 \mathrm{~min}$ and rinse with deionized water (DI) water for 1 min, and another $1 \mathrm{~min}$ in IPA, then blow the film dry with air gun. The surface cleaning process should be conducted prior to oxygen plasma treatment. Once the surface is treated, any surface touching process should be avoided in case the surface property might be changed.

\subsection{PS to PS thermopress bonding}

The thermopress bonding for PS to PS has been studied by several research groups. Our bonding process was based on the work of Young et al. [22]. The hydraulic press was preheated to $93.3^{\circ} \mathrm{C}$ for $10 \mathrm{~min}$, after which the stack was loaded and the press was closed with 6.9 MPa pressure for $60 \mathrm{~min}$. The heat was then turned off after $30 \mathrm{~min}$ to cool down in room temperature while maintaining the pressure for the rest of the time. The bonding was then complete and the PS stack was removed from the press, at which time the stack temperature was around $70^{\circ} \mathrm{C}$. This gradual release maneuver helps the bonded chip to avoid cracks, which might happen if the chip is still very hot, when unloading the bonding force.

\subsection{PS thermopress bonding strength test}

The bonding strength test was conducted with our specially designed testing devices, as illustrated in Fig.2. Air pressure was manually applied to the testing device by a syringe, and the burst pressure of the device was recorded by a pressure sensor (Vernier, Beaverton, OR) to test the tensile pressure of the PS bonding strength. 6 layers of patterned PS film were aligned and bonded to make a testing device by one thermopress bonding. The hollow space has a thickness of $0.5 \mathrm{~mm}$ (4 layers of $125 \mu \mathrm{m}$ thick film), which is large enough to guarantee a uniform pressure distribution. As shown in Fig.2 (b), when the pressure is applied from the inlet, the top and bottom bonded layers will resist the device from ballooning until the burst happens. The maximum pressure was recorded and the bonding tensile pressure can be calculated using the following equation:

$$
P_{\text {tensile }}=\frac{\mathrm{A}_{1}}{\mathrm{~A}_{2}} * P_{0}
$$

Where $P_{0}$ is the burst pressure monitored by the pressure sensor, $\mathrm{A}_{1}$ is the inner area where 
the pressure is applied on, $A_{2}$ is the ring-shape bonded area. $A_{1} / A_{2}$ is designed to be 3 so that burst can happen at low pressure.

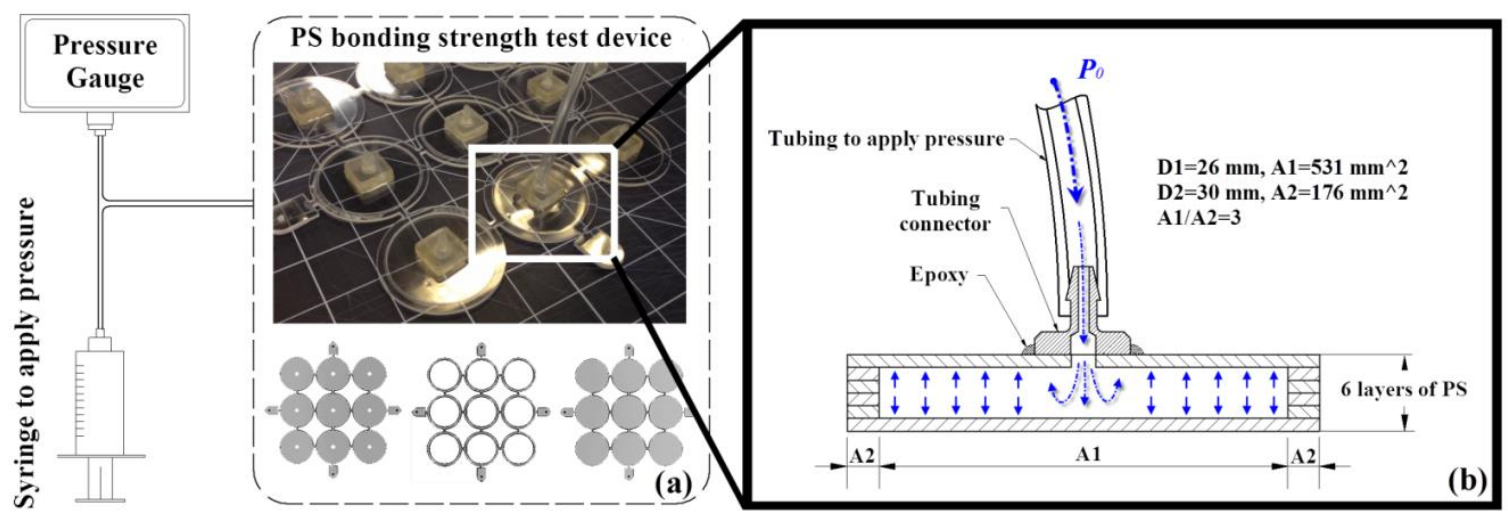

Fig. 2 PS thermopress bonding strength test. (a) Bonding strength test process. There are 9 sub-testing devices in one bonded testing device. The inlet pressure was applied from a syringe and measured by a pressure gauge. (b) Testing device schematics. The bonding strength can be characterized by the air pressure monitored to burst the device regarding the related area $\mathrm{A} 1$ and $\mathrm{A} 2$.

In this study, double cantilever method (also called the crack opening method) was also

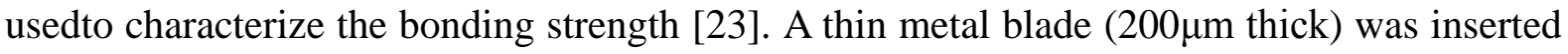
between two bonded PS films (125 $\mu \mathrm{m}$ thick), resulting in delamination. The bonding strength can be comparatively assessed by measuring the crack length with an optical microscope (AmScope, Irvine, CA). Shorter crack length means higher bonding strength.

The surface wettability of PS can be easily manipulated by oxygen plasma treatment (120 Watt, $2 \mathrm{~min}$ ), which is a major advantage of the material for microfluidics applications. However, the plasma treatment can significantly affect the thermopress bonding strength because of surface reaction due to the plasma [22]. Moreover, it is noticed during our experiments that washing the PS films using DI water after oxygen plasma treatment can affect the bonding strength. Therefore, the bonding strength tests were conducted with the two methods in several categories concerning oxygen plasma treatment and washing after the 
treatment.

\subsection{PS surface wettability manipulation}

One of the advantages of PS as microfluidic device material is that its surface wettability can be easily changed from hydrophobic into hydrophilic by oxygen plasma treatment (120 watt, Plasma Etch PE-50, Carson City, NV), and the hydrophilic surface property can last for months[24]. It is noticed that the surface wettability of PS film after plasma treatment is affected by the treatment time, as well as whether the substrate is washed by DI water after the treatment. Therefore a hydrophilicity test was conducted on PS films to study the wettability effect with different oxygen plasma treatment scenarios.

If using a shadow mask during the oxygen plasma treatment, patterned hydrophilic surface can be achieved and it can be utilized in microfluidics applications. Fig.3 presents a demonstrating example. The structure of the device is a hollow box without any geometric channels. However, the internal surface of the device is partially treated by oxygen plasma with a patterned mask, therefore a hydrophilic pattern is achieved on the inside surface of the hollow box, as is illustrated in Fig.3 (b). The shadow mask is a one-side smooth thin film performing as protection layer on the polycarbonate film (McMaster, Chicago, IL). It was applied on the PS film by electrostatic force that is strong enough for cutting and plasma treatment. The shadow mask can be removed without any residue. The film was attached to the PS surface first and then cut by the digital cutter into a patterned mask. The patterned part of the mask was then removed to expose the underlying PS film to oxygen plasma treatment. 


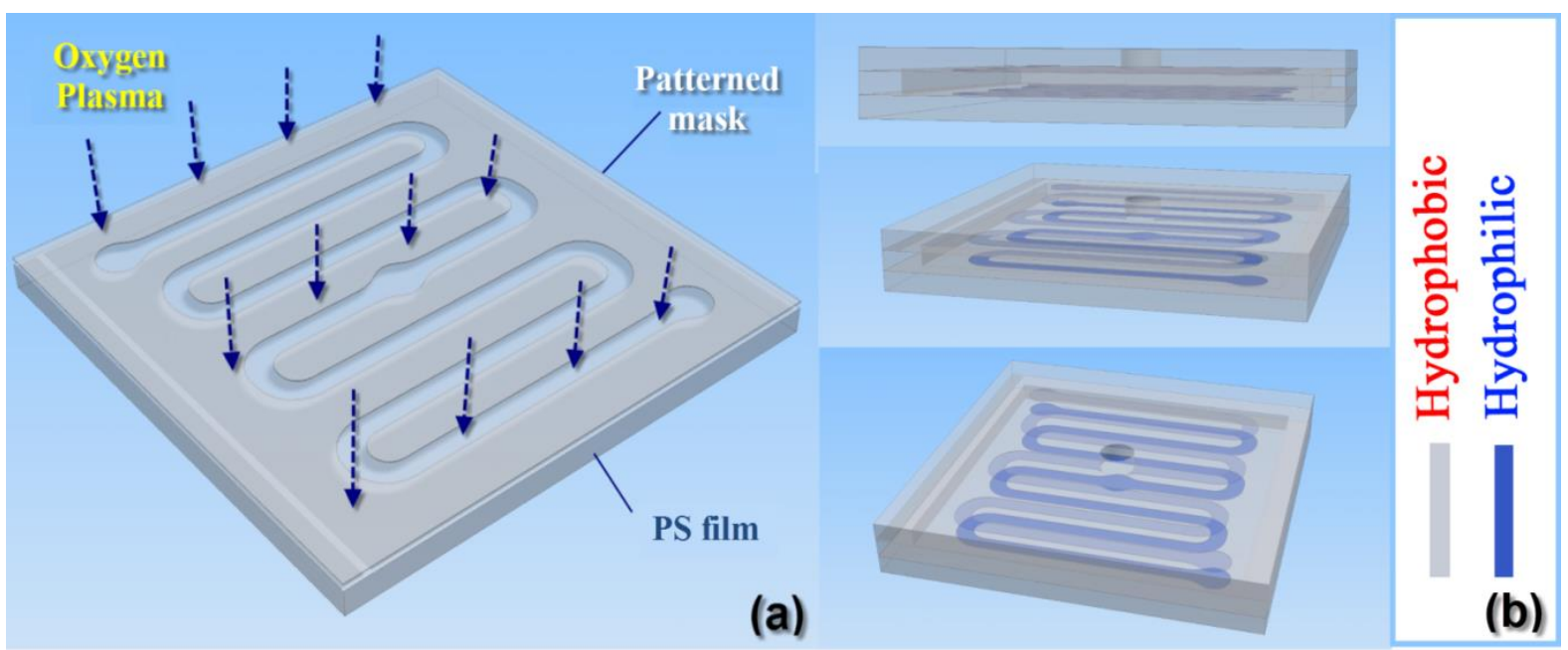

Fig. 3 PS surface wettability manipulation by oxygen plasma. (a) Partial oxygen plasma treatment achieved by a shadow mask. (b) Demonstration device with inside hydrophilic pattern.

\subsection{L-shaped passive mixer}

One of the basic applications of microfluidic device is to mix two or more reagents into one uniform solution. This can be difficult to achieve in a passive manner because the flow in microchannels is usually laminar flow which won't mix fast enough. In order to get the reagents from different inlets to mix uniformly and quickly, complicated 3D passive mixers can be implemented to achieve better mixing performance [25]. However, it is difficult to fabricate 3D microfluidic mixers compared to the 2D designs. In this study, a 3D L-shaped microfluidic mixer was used to demonstrate the capability of our microfluidic device fabrication method.

\subsubsection{Design details}

The design of the L-shaped passive mixer is shown in Fig.4. It is made of 14 layers of 125 $\mu \mathrm{m}$ PS films and it has 3D connected out-of-plane L-shaped channels from sequentially stacking and aligning all the patterned films. To form the internal 3D L-shaped channel demonstrated in Fig.4, 14 layers of $125 \mu \mathrm{m}$ PS films were used, where the first and last film perform as the top and bottom of the device. For the rest of the PS films, 4 layers with the same pattern together form the top part of the 3D channel, followed by 4 layers that form the middle part of the channel, and another 4 layers with the same pattern that form the bottom 
part (please refer to Fig.5 for better understanding). Each 4-layer part has channel thickness of $0.5 \mathrm{~mm}$, and the 3D geometry is achieved by both the pattern and the thickness of the PS films. The design process was completed in a CAD software, and the pattern of each layer was transformed to $2 \mathrm{D}$ file format for the digital craft cutter.

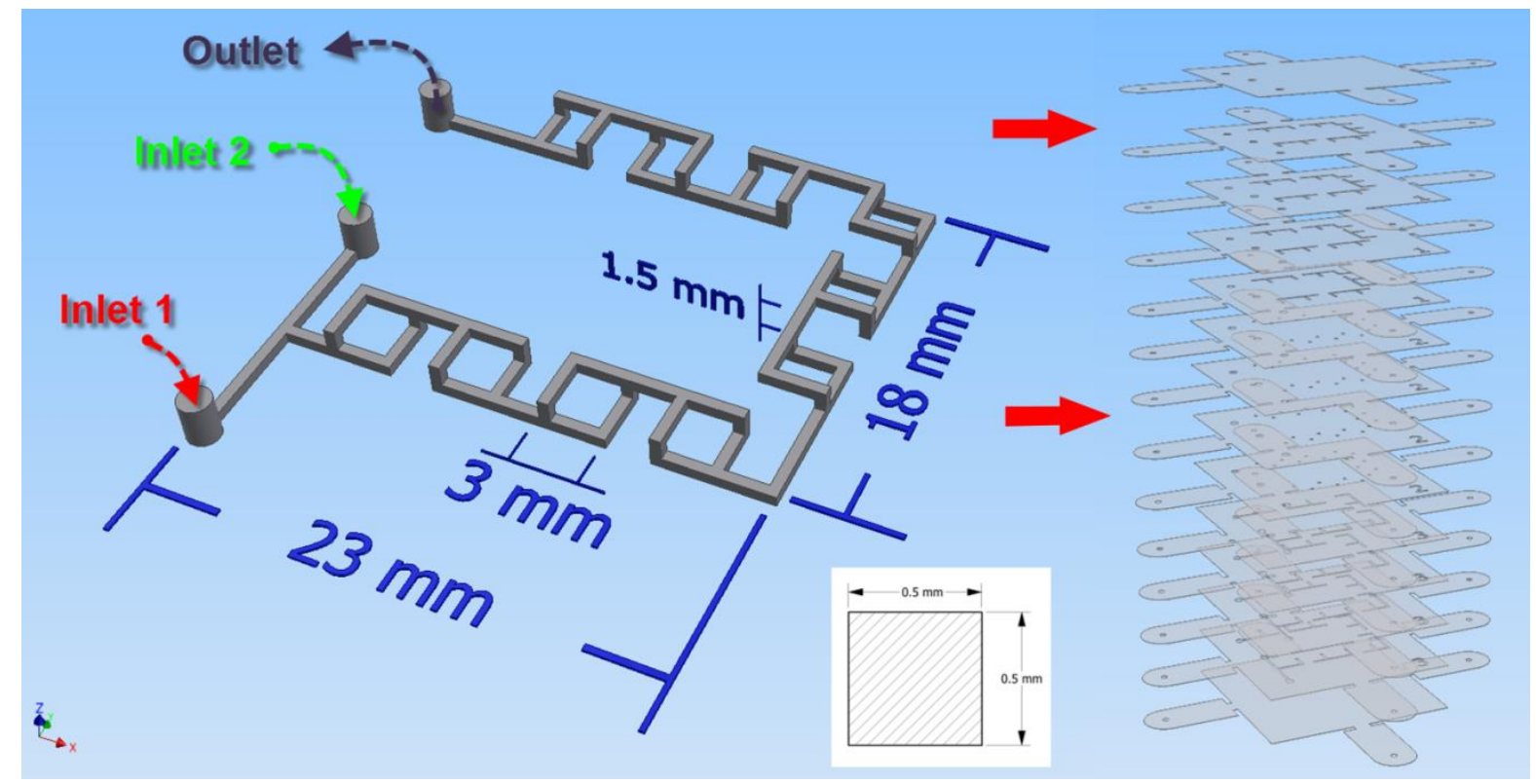

Fig. 4 Design schematic of a 3D connected out-of-plane L-shaped passive microfluidic mixer.

\subsubsection{Fabrication process}

After the film pattern was cut and prepared for each layer, the PS films are ready to be bonded together into a device. But for a complicated device with internal 3D channels like this mixer, it cannot be done by simply putting all the films together and complete the device by one thermopress bonding. The reason is that in order to achieve solid thermopress bonding, the bonded structure must not be hollow inside unless no films need to be bonded above or below the hollow feature. Therefore, to achieve solid bonding for the device, multiple steps of bonding are required (3+1), as is illustrated in Fig.5 (a). After bonding, the tubing connectors were glued on using epoxy. The finished device is shown in Fig.5 (b). 


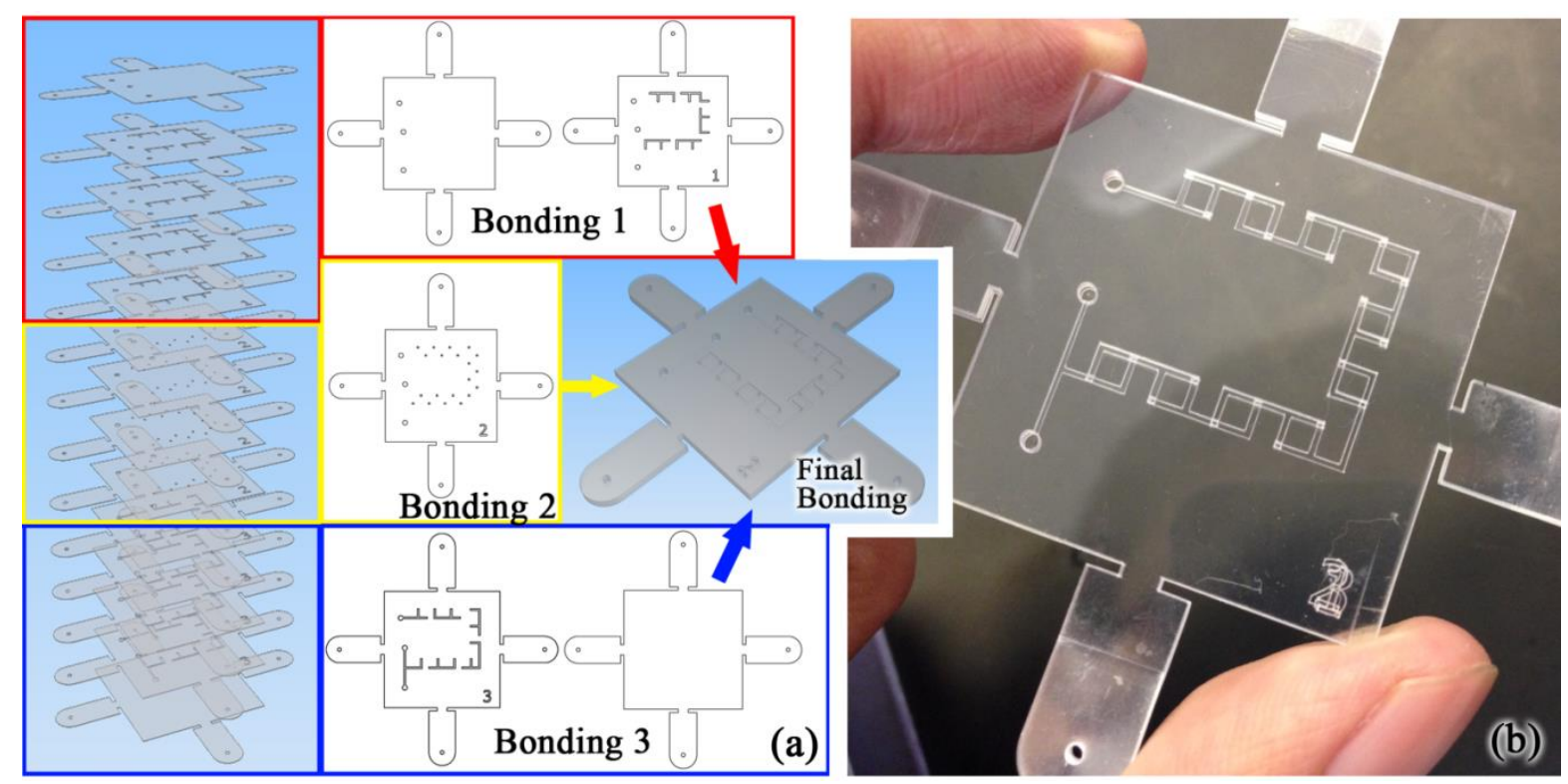

Fig. 5 Fabrication process of a 3D microfluidic mixer. (a) L-shaped 3D mixer achieved by multiple steps of thermopress bonding (three sectional bonding and one final bonding to fabricate the device). (b) Fabricated device showcase.

\section{Results and discussion}

\subsection{PS bonding strength test}

The bonding strength test results are summarized in Table 1.It is noted that the data in the table is the tensile pressure $\mathrm{P}_{\text {tensile }}$ converted from $\mathrm{P}_{0}$ using equation 1.The results show that the non-treated samples have the highest bonding strength and the value can reach as high as $375 \mathrm{KPa}$, while the fully-treated samples have the weakest bonding strength, which is lower than $50 \mathrm{KPa}$. It is also noted that the test data obtained has a large deviation, especially the non-treated category. This non-uniformity could be caused by the defects on the bonding surface, to which the test is very sensitive due to the burst mechanism. This also explains why the fully treated samples have the smallest deviation, because the oxygen plasma treatment also has surface cleaning effect [16].

Table 1 PS bonding strength results summary

\begin{tabular}{|c|c|c|c|c|}
\hline $\begin{array}{c}\text { Tensile pressure } \\
(\mathbf{K P a})\end{array}$ & $\begin{array}{c}\text { Maxbonding } \\
\text { strength }\end{array}$ & $\begin{array}{c}\text { Min bonding } \\
\text { strength }\end{array}$ & $\begin{array}{c}\text { Average bonding } \\
\text { strength }\end{array}$ & $\begin{array}{c}\text { Standard } \\
\text { deviation }\end{array}$ \\
\hline Non-treated & 375.5 & 97.5 & 200.8 & 27.5 \\
\hline
\end{tabular}




\begin{tabular}{|c|c|c|c|c|}
\hline Half-treated & 171.5 & 56.6 & 96.5 & 11.4 \\
\hline Fully-treated & 87.8 & 43.5 & 63.7 & 4.4 \\
\hline
\end{tabular}

It is shown in Table 1 that the weakest bonding case can still reach a bonding strength larger than $40 \mathrm{KPa}$, which is sufficient for most of the microfluidic devices. Because of the mechanical property of PS, the material after thermopress bonding becomes rigid which is resistive to regional failure like burst deformation. It will hold the integrity of the whole device before a total device burst failure happens, which requires much more pressure and is less likely to happen. Moreover, if stronger bonding strength is required in the device, a shadow mask can be used for the oxygen plasma treatment to protect the bonding surface while exposing the channel surface.

The above test was designed to give quantitative evaluation of PS bonding strength in several basic scenarios, where the PS films were not washed after oxygen plasma treatment. The following double cantilever method test gives a more comprehensive assessment on PS thermopress bonding under a variety of conditions. In the test, a thin blade $(200 \mu \mathrm{m})$ was inserted between two bonded PS films $(125 \mu \mathrm{m})$, and the crack length was measured to indicate bonding strength. Each of the tests was conducted on three individual samples to generate more than 20 result data. The results are shown in Table 2.

Table 2 Double cantilever method results summary

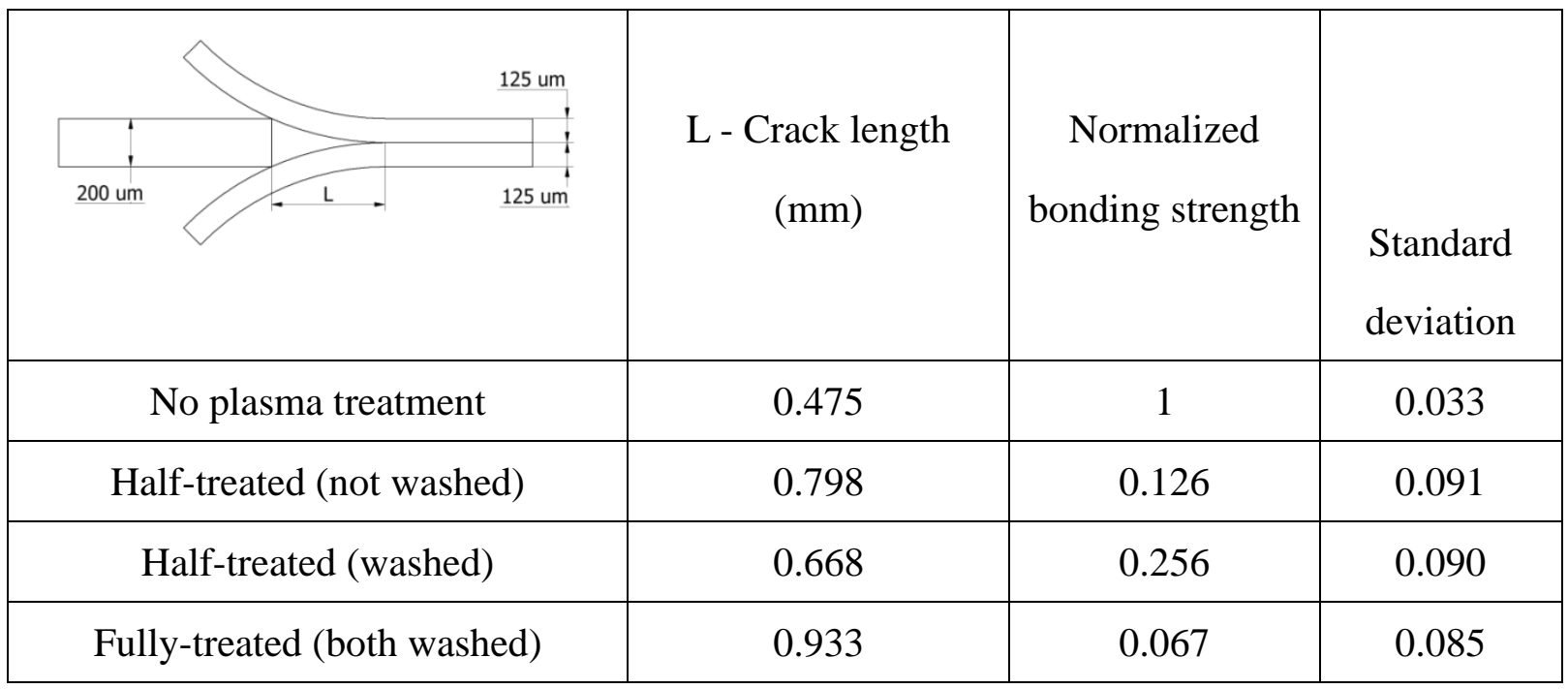




\begin{tabular}{|c|c|c|c|}
\hline Fully-treated (non-washed) & 1.020 & 0.047 & 0.048 \\
\hline Fully-treated (half-washed) & 0.993 & 0.052 & 0.082 \\
\hline
\end{tabular}

In this study, we used the double cantilever method to provide a comparative assessment for different bonding conditions. The test results have shown that oxygen plasma treatment can significantly decrease the thermopress bonding strength of PS to PS, and the fully-treated PS samples have the weakest thermopress bonding strength. This result shows the same trend with the previous bonding strength test by the chips.

To better understand and explain our results, we have conducted X-ray Photoelectron Spectroscopy (XPS) experiments to study the surface chemical change due to the effect of oxygen plasma treatment. In the experiments, we used PHI Versa Probe II instrument equipped with focused monochromatic Al K (alpha) source. Instrument base pressure was ca. $8 \times 10^{-10}$ Torr. The X-ray power of $52 \mathrm{~W}$ at $15 \mathrm{kV}$ was used for all experiments with 200 micron beam size at the X-ray incidence and take off angles of $45^{\circ}$. The instrument work function was calibrated to give a binding energy (BE) of $84.0 \mathrm{eV}$ for $\mathrm{Au} 4 \mathrm{f}_{7 / 2}$ line for metallic gold and the spectrometer dispersion was adjusted to give a BE's of $284.8 \mathrm{eV}, 932.7 \mathrm{eV}$ and of $368.3 \mathrm{eV}$ for the $\mathrm{C} 1 \mathrm{~s}$ line of adventitious (aliphatic) carbon presented on the non-sputtered samples, $\mathrm{Cu} 2 \mathrm{p}_{3 / 2}$ and $\mathrm{Ag} 3 \mathrm{~d}_{5 / 2}$ photoemission lines respectively. The PHI dual charge compensation system was used on all samples. The high resolution $\mathrm{C} 1 \mathrm{~s}$ spectra were taken with a minimum of $10-60 \mathrm{~s}$ scans using a $0.1 \mathrm{eV}$ step and $11.7 \mathrm{eV}$ pass energy. The ultimate Versa Probe II instrumental resolution was determined to be $0.15 \mathrm{eV}$ using the Fermi edge of the valence band for metallic silver. All XPS spectra were recorded using PHI software SmartSoft -XPS v2.0 and processed using PHI MultiPack v9.0. The relative sensitivity factors from MultiPack library were used to determine atomic percentage. Peaks were fitted using GL line shapes a combination of Gaussians and Lorentzians with $10-50 \%$ of Lorentzian contents. Shirley background was used for curve-fitting.

Since 2 min was the treatment time that we used in our bonding test, two PS samples 
(washed and not washed after treatment) with such treatment, alone with a reference PS sample without treatment, were tested in the C 1s XPS experiment. Fig.6 (a) shows the comparative survey spectrum of the two treated PS samples with the reference untreated PS sample. From the figure, we can notice that the intensity of Pi-Pi bond in the untreated PS sample decreased after the treatment. This indicates the broken of large polymer molecules into smaller molecules on the surface [20]. New bonds like $\mathrm{C}-\mathrm{O}-\mathrm{C}$ and $\mathrm{C}=\mathrm{O}$ were introduced to the PS surface because of the oxygen plasma treatment, which can also be observed in the Fig.6 (b, c). This explains why bonding strength was significantly reduced for oxygen plasma treated PS films, because the newly introduced surface substances have different material property with PS. Therefore the thermopress bonding temperature at $93.3^{\circ} \mathrm{C}$, which was designed according to the $\operatorname{Tg}$ of $\operatorname{PS}\left(\sim 95^{\circ} \mathrm{C}\right)$, was no longer suitable for the molecular fusion of the newly introduced substances [20]. This caused poor molecular fusion for the treated PS films with current thermopress bonding temperature, which resulted in weak bonding strength, especially for the fully treated case described above.

It is also noticed that whether the films were washed after the plasma treatment can slightly affect the bonding strength, but not enough to make significant difference. Fig.6 (b, c) and Table 3 have demonstrated data to show the surface chemical composition for the two treated PS samples, and there is a noticeable difference in carbonate, $\mathrm{C}=\mathrm{O}$, and $\mathrm{C}-\mathrm{O}-\mathrm{C}$ for the two samples. This is because during the oxygen plasma treatment, large polymer molecules on PS surface were broken into smaller molecules that were still attached to the surface, but could be easily washed away. This layer of broken small molecules on the surface will act like barrier to impede the molecular fusion process during the thermopress bonding. Therefore the washed PS samples have a slightly higher bonding strength than the non-washed ones.

As shown in Table 3, most of the carbonate has been washed away, which is due to its high solubility. The Pi-Pi bond intensity has increased after washing, which is because of the wash away of other substances like carbonate, $\mathrm{C}=\mathrm{O}$, and $\mathrm{C}-\mathrm{O}-\mathrm{C}$, that exposed more PS and therefore increased the overall detected percentage of the Pi-Pi bond. 

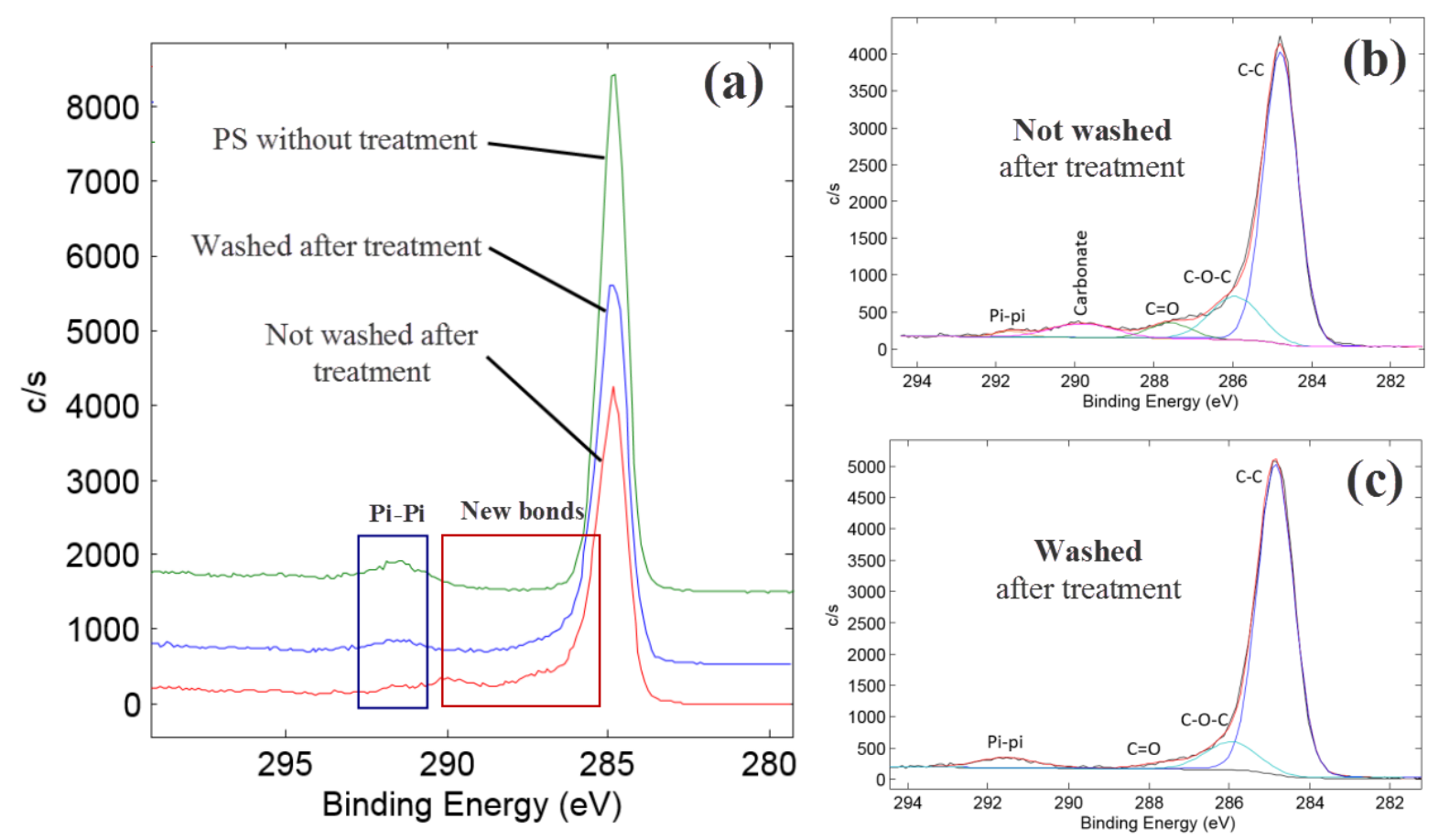

Fig. 6 C 1s XPS test on oxygen plasma treated (2 min) PS samples. (a) Comparative survey spectrum with three PS samples, it can be noticed that intensity of Pi-Pi bond decreased after treatment, and new oxygen containing bonds were formed. Comparison between (b) not washed and (c) the washed PS sample after treatment. It can be found that some oxygen containing substances could be washed away, indicating they were the loose broken molecules due to the oxygen plasma treatment. There are three curves at the peak profile that have good match with each other, they are the testing data curve, the fitting curve, and the C-C bond spectrum curve, which indicates good data reliability.

Table $3 \mathrm{C}$ 1s fit areas for PS samples with/without wash after treatment for Fig.6. Fitted values are based on \% contribution within $\mathrm{C} 1 \mathrm{~s}$ peak.

\begin{tabular}{|c|c|c|c|c|c|}
\hline & Pi-Pi & Carbonate & C=O & C-O-C & C-C \\
\hline Washed after treatment & $4.45 \%$ & $0 \%$ & $1.82 \%$ & $10.94 \%$ & $82.79 \%$ \\
\hline Not washed after treatment & $1.3 \%$ & $6.41 \%$ & $4.66 \%$ & $15.31 \%$ & $72.32 \%$ \\
\hline
\end{tabular}

However, this result is different from several previous publications. For instance, 
Bhattacharyya et al. showed that the fully plasma treated PS substrates have the highest bonding strength [26], and Young et al. showed that the fully-treated PS samples have a stronger bonding strength than the half-treated ones, yet still a lot weaker than the non-treated PS samples [22]. These controversial results on the subject could be due to the different bonding conditions (bonding recipes, facilities, PS material properties, surface treatment, etc.) that were implemented during the experiments by different research groups, which could affect the bonding strength. For instance, Bhattacharyya et al. used PS material from Pria Diagnostics (Menlo Park, CA, USA).Young et al. treated the PS surface at $60 \mathrm{~W}$ for $12 \mathrm{sec}$ and the process involves sonication $\left(10 \mathrm{~min}, 50{ }^{\circ} \mathrm{C}\right)$ and incubation $\left(37^{\circ} \mathrm{C}, 5 \% \mathrm{CO}_{2}\right)$ before bonding.

\subsection{Bonding deformation and channel resolution}

To examine the deformation after the thermopress bonding, new testing devices were designed. The testing device is made of multiple layers of $125 \mu \mathrm{m}$ thick PS film, with the patterns for measurement. The normal dimension was measured by a vernier caliper, while the transverse dimensions were measure by a microscope (AmScope, Irvine, CA).After the theromopress bonding with conditions mentioned above, the device normal thickness decreased from $1750 \mu \mathrm{m}$ to $1650 \mu \mathrm{m}(5.7 \%)$, while the transverse dimension of a pattern changed from $5000 \mu \mathrm{m}$ to $4900 \mu \mathrm{m}(2 \%)$. This means a feature dimension change around $5 \%$ can happen after thermopress bonding, which will affect the overall resolution of the device. It is noted that if high precision is required, the dimension adjustment should be considered into the design to compensate the deformation.

The following examination is about the channel resolution of the microfluidic devices achieved by this fabrication method. Fig.7 shows the SEM images of the cross-section view of the microchannels achieved with our method. A wide channel $(125 \mu \mathrm{m} \times 700 \mu \mathrm{m})$ is shown in Fig.7(a) with very little channel collapse or deformation. This desirable result is attributed to the high stiffness of the pre-bonded top and bottom capping layers of the channel, because thermopress bonding process greatly increases rigidity of the material [14]. Fig.7(b) shows a channel cross-section view with a dimension of $125 \mu \mathrm{m} * 125 \mu \mathrm{m}$, which is the resolution for 
this thickness to come up with decent microchannels. It is believed that with thinner PS films (as low as $25 \mu \mathrm{m}$ that is commercially available), even higher channel resolution might be achieved.

The resolution of the device is also affected by the alignment tolerance in case of multiple layers bonding, which in turn is determined by the film pattern itself (decided by the digital cutter's tolerance, currently $25 \mu \mathrm{m}$ ) as well as the alignment method. It was examined under SEM that the alignment tolerance of our current method is around $35 \mu \mathrm{m}$, which is good enough for our current microfluidic devices whose channel width is around $1 \mathrm{~mm}$. If higher alignment tolerance is needed for better device resolution, other more sophisticated alignment method can be implemented.
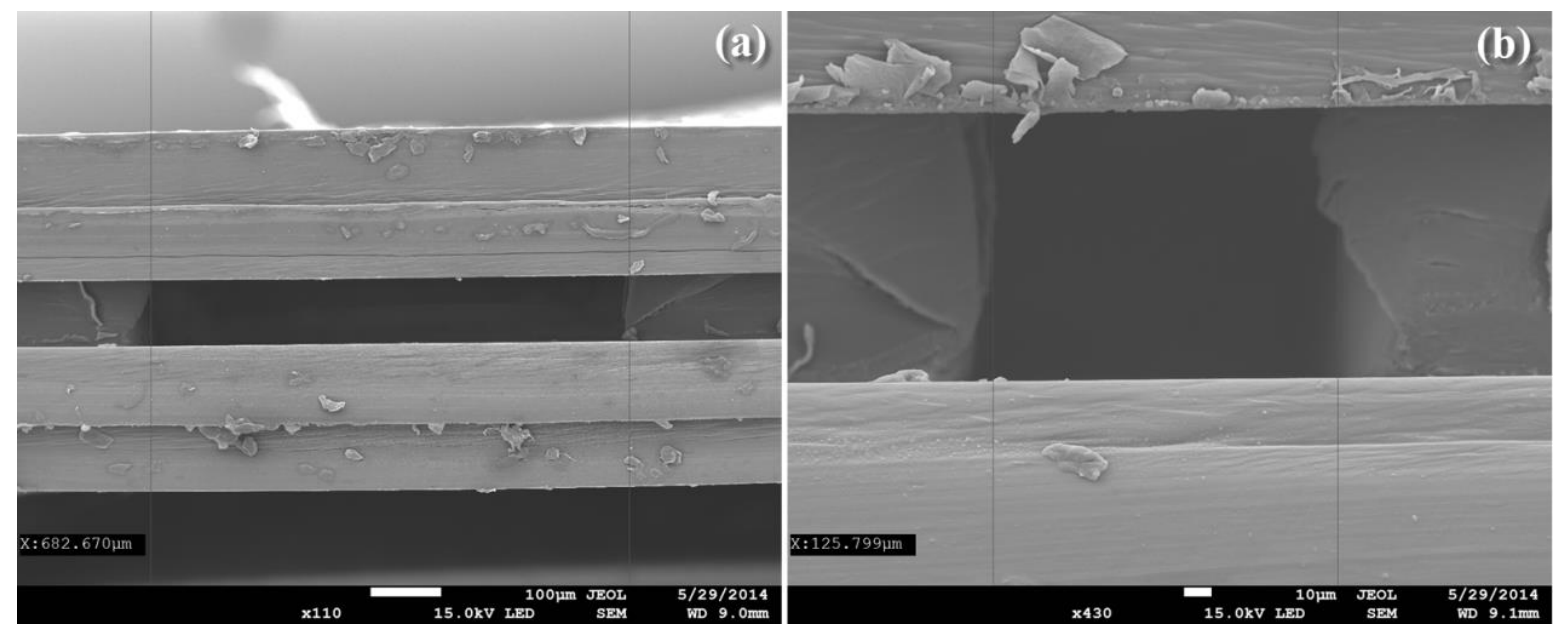

Fig. 7 SEM images of channel sectional view for resolution and deformation examination. (a)

Channel width is $700 \mu \mathrm{m}$, channel height is the thickness of 1 layer of PS film, $125 \mu \mathrm{m}$. (b)

The smallest microfluidic channel achieved with this method is $125 \mu \mathrm{m} * 125 \mu \mathrm{m}$.

\subsection{PS surface wettability manipulation}

A surface wettability test was conducted to study the PS surface wetting with different oxygen plasma treatment time. Fig. 8 shows the testing result by measuring the contact angle, which is obtained by a goniometer (ramé-hart, Succasunna, NJ). As shown in Fig.8, the PS films used in the experiment are hydrophobic in nature and have a contact angle of $93^{\circ}$. After the oxygen plasma treatment (120 watt, Plasma Etch PE-50), the measured contact angle 
decreased dramatically, making the surface hydrophilic. It is noticed that the measured contact angle of the washed samples is a little higher than the non-washed samples, which can be explained by the same reason in the bonding strength test. Generally, longer plasma treatment time results in lower contact angle. The contact angle tends to stabilize after 4 min of treatment. The stabilized contact angle approximates 25 degrees for the washed sample, and 18 degrees for the unwashed sample. This contact angle difference for the two cases can be explained by the results shown in Table 3. The unwashed PS sample has more oxygen containing substance on the surface than the washed PS sample, which is the reason it is more hydrophilic.

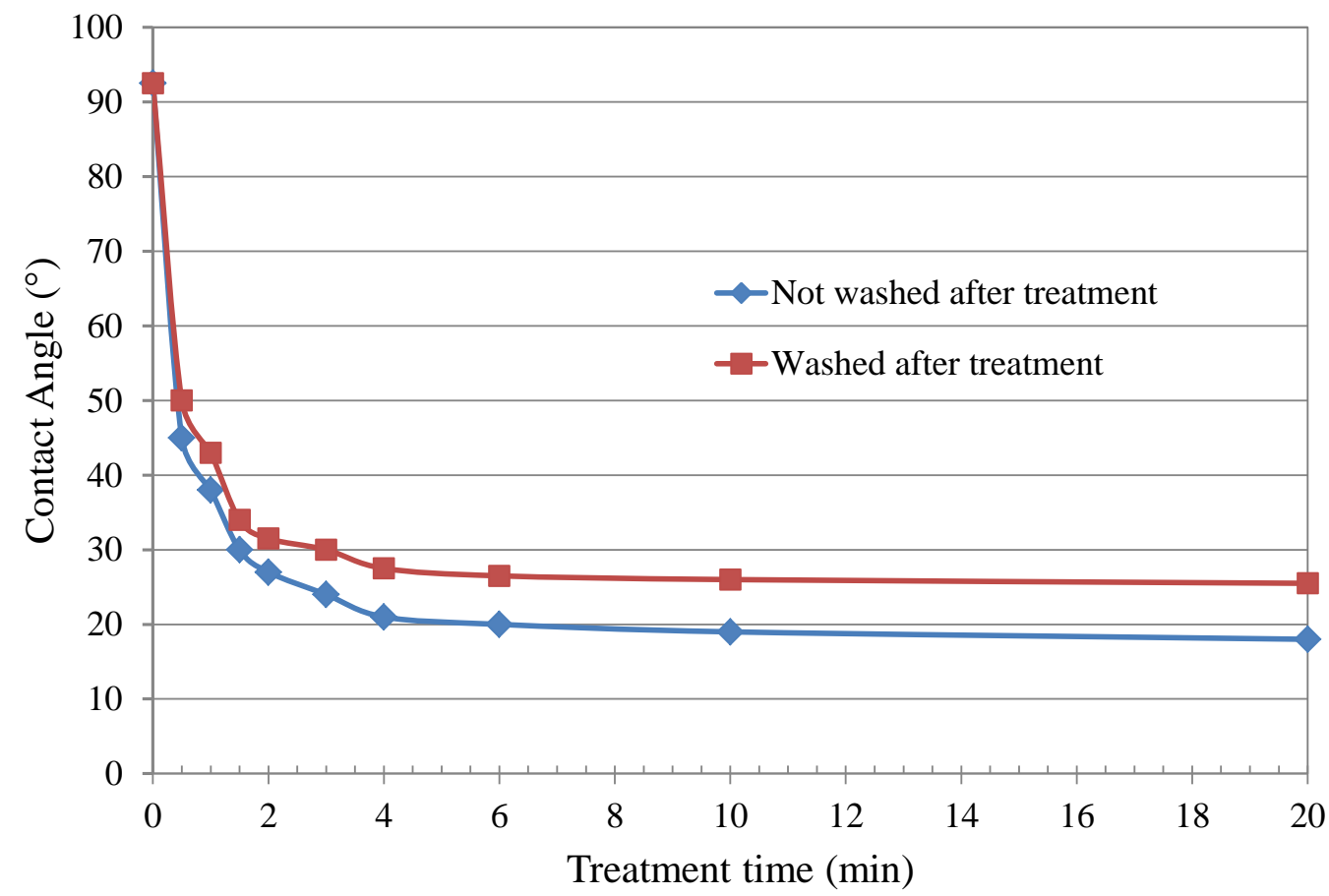

Fig. 8 PS surface wettability testing result. Longer treatment time results in smaller contact angle, and the value tends to be stabilized after 4 min of treatment with oxygen plasma (120 watt). It is also noted from the result that washing the samples after plasma treatment can slightly increase the contact angle.

Since the surface wettability of PS can be easily manipulated, when using a patterned shadow mask during the oxygen plasma treatment, a patterned hydrophilic surface can be created. The device shown in Fig.9 is an example to utilize this surface manipulation property 
as is explained in the experimental part. Because of this regional patterned wettability, when the green liquid was pumped from the inlet at a rate of $20 \mu \mathrm{L} / \mathrm{min}$ by a syringe pump (KD Scientific, Holliston, MA), the green solution went through the pattern instead of spreading randomly even though there is no structural channel inside the device.
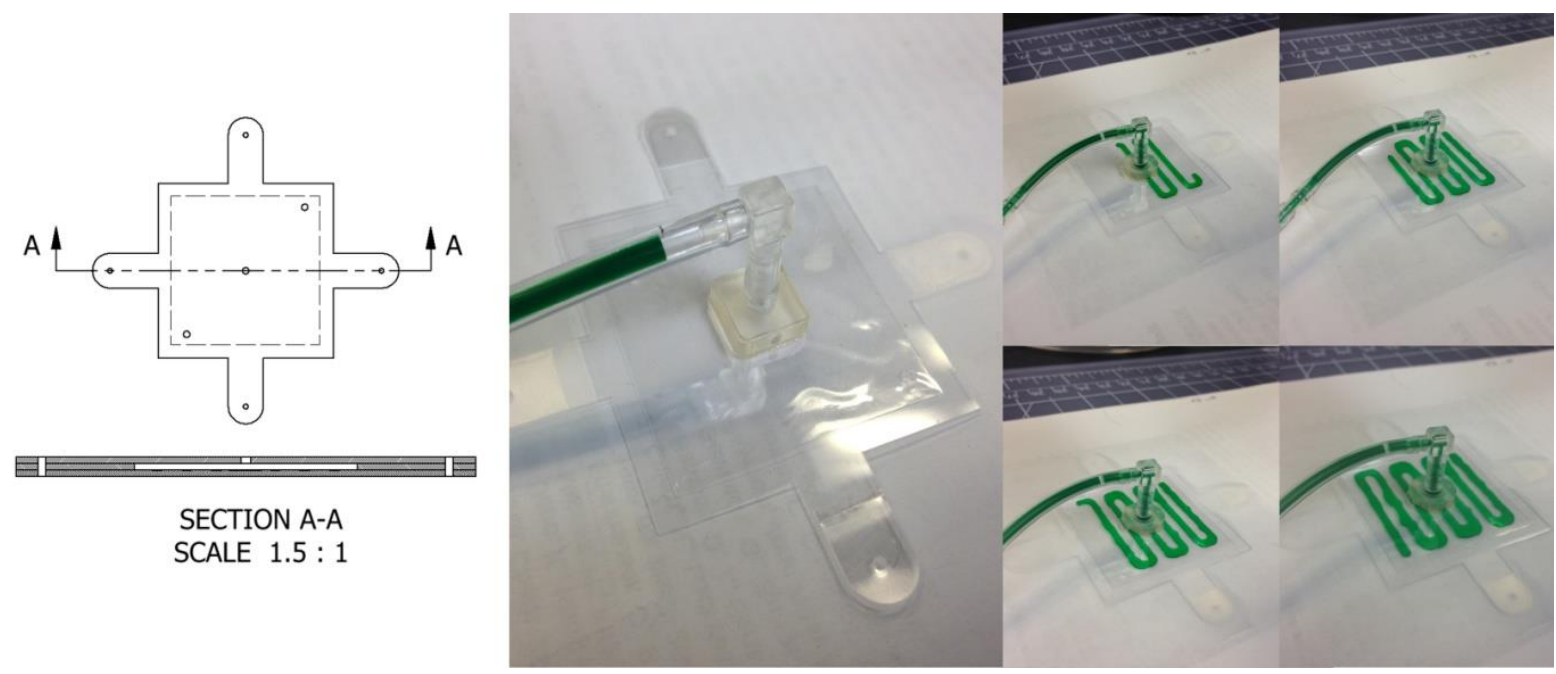

Fig. 9 PS surface wettability manipulation demonstration. Since the inside surface of the testing device is partially treated by oxygen plasma to be hydrophilic, the injected green solution travels through the pre-treated hydrophilic pattern even though there is no physical channel in the hollow structure.

\subsection{L-shaped 3D passive mixer}

To test the device, we used a syringe pump to simultaneously pump red and green solutions from the inlet at a flow rate of $50 \mu \mathrm{L} / \mathrm{min}$. As shown in Fig.10, the two different colored solutions are optically observed to be fully mixed at around $20 \mathrm{~mm}$ after they entered the device. Given that the sectional area is $0.25 \mathrm{~mm}^{2}$, and the combined flow rate of the solution is $100 \mu \mathrm{L} / \mathrm{min}$, which means it only takes $3 \mathrm{~s}$ for the solutions to mix in the device once entered. The reason of this good mixing result is explained in Fig.10(f). Because in the 3D microchannel design, the two reagents are split into two streams and forced to join together, and this agitating passive behavior is continuously repeated which greatly promotes mixing effect. If the splitting and joining junctions can be made closer to each other $(3 \mathrm{~mm}$ in current device), the mixing efficiency could be increased significantly. It is also worth 
mentioning that the bonding method has very high yield, about $90 \%$ for single step two-layer bonding, and almost 100\% for multiple layers bonding. The occasional bonding failure region only appears at the edge of the bonded chip, where no important features are usually designed. Moreover, the bonding failure region can easily be repaired by repeated bonding during the following multiple layer bonding process.

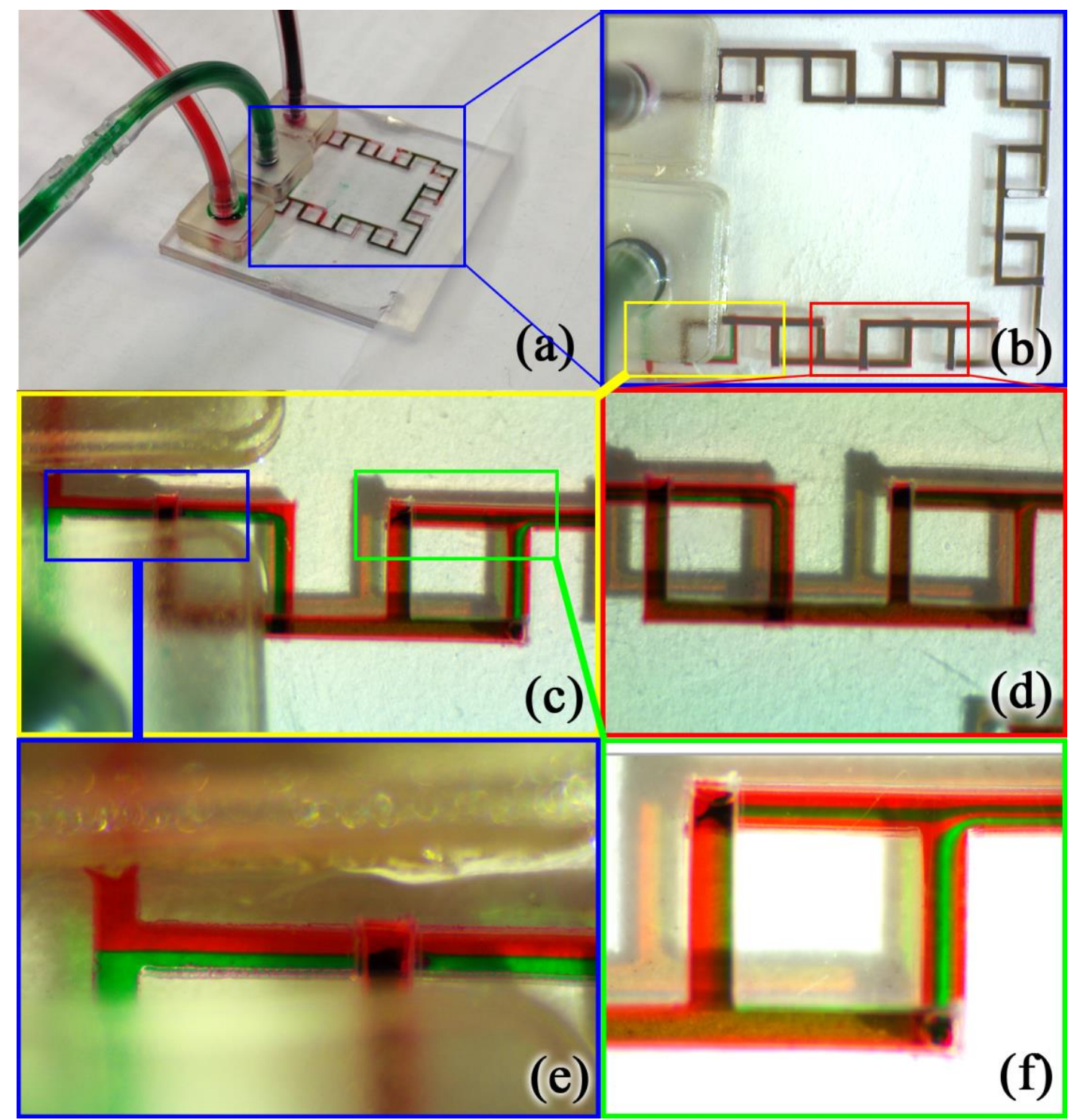

Fig. 10 Testing result of the 3D L-shaped microfluidic mixer. (a,b,c) Mixer test demonstration.

(d) Two colored reagent mixed uniformly after entering the device in about $10 \mathrm{~mm}$. (e)

Laminar flow in the T-shape entrance results in poor mixing. (f) Repeated splitting and emerging the reagents greatly promotes mixing. 


\section{Conclusions}

In this paper, we have introduced a device fabrication method for microfluidics applications and conducted a series of experiments to test its validity. The bonding strength tests have shown bonding tensile pressure of the device ranging from $43.5 \mathrm{KPa}$ to $375.5 \mathrm{KPa}$, which is sufficient for microfluidics applications. The bonding deformation check from the SEM images showed very little channel collapse. PS surface wettability manipulation has shown controllable device surface property, that has significant meaning for microfluidics applications. And finally, the successful test of the 3D L-shaped microfluidic mixer validatesour method to fabricate 3D microfluidic devices from PS films. This bonding method has very high yield, about $90 \%$ for single step two-layer bonding, and almost $100 \%$ for multiple layers bonding.

The merit of this method is that it provides a cheap, fast, and relatively accurate way to fabricate microfluidic devices. The equipment needed during the process is a digital craft cutter and a hydraulic hot press. The primary device material is PS, which is commonly available. Once the design is done, it only takes several hours to fabricate a device, including film material preparation and thermopress bonding. The feature resolution of this method is limited by both the pattern resolution from the digital craft cutter $(25 \mu \mathrm{m})$ and the film thickness, which can be found commercially available to be as low as $25 \mu \mathrm{m}$. Therefore, we believe that this method is capable of a variety of microfluidics applications, and it is especially helpful to scientific lab researchers who constantly need to change their designs.

\section{Acknowledgement}

This material is based upon work supported by the National Science Foundation under Grant No. 1264739. We would like to acknowledge the Integrated Nanosystems Development

Institute (INDI) at IUPUI for use of their shared facilities (craft cutter, FESEM, etc.) in support of our research. We would also like to thank the Molecular Structure Center at Indiana University Bloomington (IUB), and especially the XPS facility supervisor Dr. Yaroslav Lozovyy, for his help on our XPS experiments. 


\section{Reference}

[1] Teh S-Y, Lin R, Hung L-H and Lee A P 2008 Droplet microfluidics Lab on a Chip 8 198-220

[2] Dittrich P S and Manz A 2006 Lab-on-a-chip: microfluidics in drug discovery Nature Reviews Drug Discovery 5 210-8

[3] Gravesen P, Branebjerg J and Jensen O S 1993 Microfluidics-a review Journal of Micromechanics and Microengineering 3168

[4] Beebe D J, Mensing G A and Walker G M 2002 Physics and applications of microfluidics in biology Annual review of biomedical engineering 4 261-86

[5] Stone H A, Stroock A D and Ajdari A 2004 Engineering flows in small devices: microfluidics toward a lab-on-a-chip Annu. Rev. Fluid Mech. 36 381-411

[6] Squires T M and Quake S R 2005 Microfluidics: Fluid physics at the nanoliter scale Reviews of modern physics 77977

[7] Duffy D C, McDonald J C, Schueller O J and Whitesides G M 1998 Rapid prototyping of microfluidic systems in poly (dimethylsiloxane) Analytical chemistry 70 4974-84

[8] Anderson J R, Chiu D T, Wu H, Schueller O J and Whitesides G M 2000 Fabrication of microfluidic systems in poly (dimethylsiloxane) Electrophoresis 21 27-40

[9] Océane L, Mariotti M, Yang M, Hall G N and Chiang D The Study of Laminar Flow

[10] Kim P, Kwon K W, Park M C, Lee S H, Kim S M and Suh K Y 2008 Soft lithography for microfluidics: a review

[11] McDonald J C and Whitesides G M 2002 Poly (dimethylsiloxane) as a material for fabricating microfluidic devices Accounts of chemical research 35 491-9

[12] Shatford R and Karanassios V 2014 Microplasma fabrication: from semiconductor technology for 2D-chips and microfluidic channels to rapid prototyping and 3D-printing of microplasma devices. In: SPIE Sensing Technology+ Applications: International Society for Optics and Photonics) pp 91060H-H-8

[13] Yuen P K and Goral V N 2010 Low-cost rapid prototyping of flexible microfluidic devices using a desktop digital craft cutter Lab on a Chip 10 384-7

[14] Fan Y, Li H, Yi Y and Foulds I G 2014 PMMA to Polystyrene bonding for polymer based microfluidic systems Microsystem Technologies 20 59-64

[15] Tang L and Lee N Y 2010 A facile route for irreversible bonding of plastic-PDMS hybrid microdevices at room temperature Lab on a Chip 10 1274-80

[16] Reymond F, Rossier J S and Girault H H 2002 Polymer microchips bonded by O2-plasma activation Electrophoresis 23 782-90

[17] Tsao C-W and DeVoe D L 2009 Bonding of thermoplastic polymer microfluidics Microfluidics and Nanofluidics 6 1-16

[18] Wang Y, Balowski J, Phillips C, Phillips R, Sims C E and Allbritton N L 2011 Benchtop micromolding of polystyrene by soft lithography Lab on a Chip 11 3089-97

[19] Yuen P K and DeRosa M E 2011 Flexible microfluidic devices with three-dimensional interconnected microporous walls for gas and liquid applications Lab on a Chip $\mathbf{1 1}$ 3249-55

[20] Grace J M and Gerenser L J 2003 Plasma treatment of polymers Journal of dispersion science and technology 24 305-41 
[21] Chen C S, Breslauer D N, Luna J I, Grimes A, Chin W C, Leeb L P and Khine M 2008 Shrinky-Dink microfluidics: 3D polystyrene chips Lab on a Chip 8 622-4

[22] Young E W, Berthier E, Guckenberger D J, Sackmann E, Lamers C, Meyvantsson I, Huttenlocher A and Beebe D J 2011 Rapid prototyping of arrayed microfluidic systems in polystyrene for cell-based assays Analytical chemistry 83 1408-17

[23] Vallin Ö, Jonsson K and Lindberg U 2005 Adhesion quantification methods for wafer bonding Materials Science and Engineering: R: Reports 50 109-65

[24] Larsson A and Derand H 2002 Stability of polycarbonate and polystyrene surfaces after hydrophilization with high intensity oxygen RF plasma Journal of Colloid and Interface Science 246 214-21

[25] Suh Y K and Kang S 2010 A review on mixing in microfluidics Micromachines 1 $82-111$

[26] Bhattacharyya A and Klapperich C M 2007 Mechanical and chemical analysis of plasma and ultraviolet-ozone surface treatments for thermal bonding of polymeric microfluidic devices Lab on a Chip 7 876-82 